\title{
Case report of speech- language therapy work for voice adequacy of a male transgender
}



\begin{abstract}
The research objective was to present a speech-language therapy intervention for voice readjustment of a male transgender after the use of hormones. We will initially approach the issue from the social and biological perspectives, besides presenting the process of hormonal intervention. Subsequently, we will explain the role of Speech-Language Therapy in the vocal readjustment process, demonstrating the phases of care performed in the vocal readjustment project of the Veiga de Almeida University transgender public. Acoustic analysis of the voices of two transgender men before and after speech-language therapy will be presented. For this, speech samples of sentences were used.
\end{abstract}

\section{Keywords: speech acoustics, transgender people, voice quality}

\section{Introduction}

Sex is a biological appointment that denominates people according to their genitalia, i.e., their organic features. Therefore, Lanz [1] presents four types of "sex": 1) male: an individual who is born with a penis; 2) female: an individual who is born with a vagina; 3) intersexual: an individual who is born with an erroneous combination of penis and vagina; 4) null: individual who does not have any genital trait.

Gender is the social classification of the human being, which can be a man or a woman, and gender role is everything we consider culturally as male or female. For the same author [1], gender is a social construction, which differs between cultures and eras. In the Western culture, there is only male and female, which should base on the characteristics of the genitalia, in which the gender binary shows that people born male and female are men and women, respectively. Some people are born with female biological sex but identify them with the male gender, or vice versa. According to Avila and Grossi [2], a transgender is a person who does not feel like belonging to the biological sex. For the authors, a male transgender is an individual biologically born female but self-identifies, through nomination, clothes and body changes, as belonging to the male gender. According to Nery and Maranhão [3], the beginning of the feeling of strangeness with the body and with the identity usually starts in childhood. Transsexuality was classified as a disease according to the Brazilian contexts, which required regulation of services specialized in the care for this group [4].

Starting from the idea that a transsexual person was the bearer of "psychological deviance" and that could put his own life at risk, in 1980, the Federal Council of Medicine authorized, experimentally and for research purposes, transgenitalization surgeries. This fact occurred after the council received processes focused on the transsexuality issue, coming from health professionals and institutions concerned with the need for a medical intervention to meet a group who lived in great suffering. In the same year, the term transsexual was inserted in the Diagnostic and Statistical Manual of Mental Disorders, $3^{\text {rd }}$ Edition (DSM-III) and transsexuality was defined as a syndrome of "gender dysphoria" for those with dysphoric gender demonstrating, for at least two years, a constant yearning to reassign their genital sex and gender change [5].

In 1993, the World Health Organization (WHO) put transsexuality as a disorder of sexual identity, as inserted in the International

\author{
João Carlos Lopes ${ }^{1 *}$, \\ Katia Alves Da Silva ${ }^{1}$, \\ Renata Vieira², \\ Viviane Dos Santos \\ Marques $^{1}$, Léslie \\ Piccolotto Ferreira ${ }^{3}$, \\ Marta Assumpção \\ De Andrada E Silva ${ }^{3}$, \\ Reynaldo Gomes Lopes ${ }^{1}$ \\ and Monica Azzariti ${ }^{1}$ \\ ${ }^{1}$ Veiga de Almeida University, Brazil \\ ${ }^{2}$ Fluminense Federal University, Brazil \\ ${ }^{3}$ Pontifical Catholic University of São \\ Paulo, Brazill \\ *Author for correspondence: \\ joao.lopes@uva.br
}


Classification of Diseases (ICD-101). Therefrom, it began to be seen as a disease due to the insertion of the suffix "ism". In 1994, with the launch of the DSM-IV, the expression "transsexualism" is replaced by "Gender Identity Disorder", which can also be found in the ICD$10[5]$.

From 1997, the surgical interventions for sex reassignment process came out from clandestineness and were initiated legally in the country through Resolution 1,482/97 of CFM, in which the neophalloplasty or any other supplementary procedure on gonads or secondary sexual characters would be carried out experimentally and only in university hospitals or people adequate to the research.

In August 2008, the transsexualism process was established through ordinances 1,707 and 457 , which should be held at the UHS level, ensuring integrity, humanization in the procedure, quality of the transsexualism process for the transsexual, empowerment of teams, user embracement, use of the social name and therapeutic follow-up with access from harmonization until sex reassignment surgery.

In 2013, the Ministry of Health published Ordinance 2,803, which repealed Ordinance $1,707 / 08$, redefining and broadening the transsexualism process in the UHS, presenting some ideas, such as the Health Care Network $(\mathrm{HCN})$ and healthcare lines. The Ministry also implemented the clinic for transsexuals, launches the follow-up in the pre- and postoperative phases and requires the hormone treatment in the transsexualizer process and for the preparation of sex reassignment surgery. After this publication, there have been negative reports in relation to the provided assistance; according to Lima and Cruz [4], there is a small interaction with other UHS services and, according to reports of interviews conducted by the authors, the interlocutors reported a trivialization of suffering and lack of commitment with the period of the interventions.

For Saadeh [6], the use of testosterone increases sexual desire and the social interests of the individual. It brings more willingness and some of them mention feeling more aggressively. There is also weight increase, reduced hip fat and greater strength in upper limbs. Characteristics considered male, such as beard and hair growth, bass voice, reduced adipose tissue, which acquires male characteristics and the appearance of the muscle tissue arise and improve the selfesteem of these men. Some organic attributes are differentiated, such as increased clitoris and atrophy of the breasts.

According to Harry Benjamin International Gender Dysphoria Association-HBIGDA, the process of hormone intervention has an important role in relation to the individual's anatomy and psychic, improving his mental health and quality of life, because he feels alike the gender of affinity.

For Saadeh [6], the beginning of the process of hormone intervention should follow some criteria, such as the person must be at least 18 years old, must have a considerable understanding about the hormone that will be used, in addition to having in minded its risks and privileges. The person must also have at least three months of experience of life change in the desired sex or a period in treatment with psychologists with duration established by them prior to the beginning of the hormone intervention.

Saadeh [6] affirms that the reassignment surgery is not synonymous to the end of the treatment; for transsexuals, this procedure is the accomplishment of a desire, although it is only an anatomical change. A post-surgery follow-up is necessary to give continuity to the treatment.

From the dissemination of laws, and more precisely Ordinance 457, of August 2008, which included the speech-language therapist in the multidisciplinary team of the transsexualizer process, several specialized services were created for the transgender public. Including the speech-language therapist in the team is of great importance because this professional will bring the individual's voice to the gender of affinity. The voice has great social importance and each person has a distinct voice, which transmits part of the personality; it shows aspects related to the psychological and emotional condition and spreads a meaning beyond the content of the speech [7].

Speech-Language therapy is important for a new awareness of the vocal tract, and promoting sound effort is not always enough to characterize the voice in the reported gender. In this therapeutic proposal, the patient will hear his voice during the appointment, will have 
new ways to use it, thus, with this "new voice", the individual will create various forms to live and relate with society and with himself [7]. The biological factors, social pressure, means of communication and cultural patterns are elements that influence vocal images of people [8].

Jacobs [9] shows that even, with the vocal parameters in studies, the fundamental frequency can be a way to distinguish between male and female voices. Anyone can differentiate a "woman's voice" from a "man's voice" and, due to this, many transsexuals are seeking speechlanguage offices. The theories that anchor the present research are the Model of Phonetic Description of Voice Quality and Acoustic Theory of Speech Production.

The Model of Phonetic Description of Voice Quality (LAVER) understands voice quality as the result of a set of articulatory and sound adjustments used by the speaker and which show the individual muscle trend of the configuration of the vocal tract. From this theoretical reference, the VPAS guide emerged, which was adapted to the Brazilian reality by Camargo [10]. The present study aims to describe the process of speech-language intervention for voice adequacy of a male transgender patient by means of samples of phrases recorded using two types of analysis: auditory-perceptual and acoustic, seeking to correlate the findings and contribute to a reflection on the population of transgender people who increasingly need speech-language actions each day.

\section{Method}

The Ethics Committee of the Veiga de Almeida University approved this research under number 2.376.533. The research subject voluntarily agreed to participate in the study and signed the Informed Consent Form. This is a case study. Here, we present the aspects related to materials and methods of analysis for the development of this case study. These aspects include the history of the evaluated patient, the materials and procedures for the collection of speech samples and data analysis.

Since March 2016, the Health Center of the Veiga de Almeida University, in Rio de Janeiro, has offered free therapy for voice adequacy to the transgender public. The goal of this project is to contribute to the modification of the vocal characteristics of the transgender population, taking into account the different ages and clinical conditions presented, seeking to adapt their voice to their gender identity, promoting the insertion of this population into social groups and labor market.

\section{- Case description}

L.H.P., 33 years old, Physical Education Teacher, sought the aforementioned outpatient clinic with the following complaint: " $M y$ voice bothers me a lot" (SIC). According to the patient, his voice is not in the same tone, it is uncontrolled and unexpectedly "untunes". In relation to vocal health LHP denies smoking and reports drinking socially. His last nasofibroscopic test revealed polyps in the left nasal cavity; he takes medicines to treat gastroesophageal reflux disease and complained of frequent cough and phlegm. The patient reported that, when talking on the phone, people call him madam, even after saying his social name. His expectation is "to have a deeper voice not to be confused with a woman's voice” (SIC).

The speech-language evaluation showed higher, mixed breathing, pneumophonicarticulatory incoordination, and medium to a high pitch, adequate loudness, laryngopharyngeal resonance, and speed of speech median to slow, precise articulation and vocal quality with slight tension in moments of shrill exacerbations.

The patient was worked on the vocal guidance and awareness, deepening of the fundamental frequency, phonation in smile and articulatory sharpness, exploitation of different emissions and ascending or descending modulation at the end of emissions, modification of the parameters of intonation, resonance, in vocabulary, pronunciation, in the linkage and the physiognomic and expressive gestures during speech. In the nasofibrolaryngoscopy examination performed by an otorhinolaryngologist, the patient's vocal folds showed no alterations. When he arrived for the treatment, he had made use of the duration hormone for three years before starting the phonotherapy.

\section{- Procedures}

For a collection of the samples before and after therapy for voice adequacy, the recording 
was performed with a Zoom H5 portable recorder with a built-in microphone positioned at a distance of 60 centimeters from the mouth of the subject. The recordings were performed at the sampling frequency of $44,100 \mathrm{~Hz}, 16$ bit and .wav extension. For the sample, the sentences of the CAPE-V were used in two moments before the beginning of the sessions and after 15 weeks of care.

- Erica drank a pear and blackberry juice

- Sonia can dance the samba alone

- Look at that blue airplane

- Now it's time to finish

The auditory-perceptual analysis was performed by a speech-language therapist with phonetic training and four-year experience in the implementation of the VPAS guide. The files with samples of phrases recorded before and after the therapy were sent to the judge, who performed the task of tracing the profiles of voice quality.

The acoustic analysis was performed at the free software PRAAT [11] available on http:// www.fon.hum.uva.nl/praat/. The collected audios were initially equalized through the program Sound Forge Pro 11, subsequently opened in the Praat program and segmented, being the vowels separated for inspection of their duration.

To verify prosodic and paralinguistic aspects, the following parameters were analyzed for long and short term analysis:

- average, minimum and maximum fundamental frequency

- average, minimum and maximum intensity

- Duration of vowels

\section{Results}

The results of the auditory-perceptual analysis showed a significant difference in the pattern of voice quality of the subject, pre and post vocal therapy. Regarding the vocal tract and sound aspects, we observed that the subject's voice profile, after the therapy, was closest to the neutral adjustment. Regarding aspects of vocal dynamics, we can observe a difference in the pitch characteristics, whose extent and variability reduced, and the difference in the elocution rate, which went from slow to fast.
The acoustic analysis showed long- and shortterm parameters. The long-term parameters extracted were F0 measures and intensity, and the results are in the tables below:

TABLES 1-4 show that, regarding the extraction of F0 measures in four sentences, pre and post-speech-language therapy, and both the extension as the maximum frequency decreased after the therapy. This result demonstrates that both the extension as the pitch variability decreased, thus showing changes in the intonation contour of the sentences.

Below, TABLES 5-8 refer to the extraction of measures of intensity in the four sentences, pre and post speech-language therapy:

\begin{tabular}{|c|c|c|}
\hline Fundamental frequency (hz) & Pre-therapy & Post-therapy \\
\hline Minimum & 80 & 79 \\
\hline Maximum & 262 & 156 \\
\hline Average & 135 & 121 \\
\hline
\end{tabular}

TABLE 2. Analysis of minimum, maximum and average $F 0$ values, in the sample of the sentence "Sonia can dance samba alone", collected pre and post speech-language therapy of voice adequacy.

\begin{tabular}{|c|c|c|}
\hline Fundamental frequency (hz) & Pre-therapy & Post-therapy \\
\hline Minimum & 79 & 80 \\
\hline Maximum & 223 & 139 \\
\hline Average & 110 & 113 \\
\hline
\end{tabular}

TABLE 3. Analysis of minimum, maximum and average F0 values, in the sample of the sentence "Look at that blue airplane", collected pre and post speech-language therapy of voice adequacy

\begin{tabular}{|c|c|c|}
\hline Fundamental Frequency $\mathbf{( H z )}$ & Pre-therapy & Post-therapy \\
\hline Minimum & 82 & 91 \\
\hline Maximum & 254 & 206 \\
\hline Average & 119 & 118 \\
\hline
\end{tabular}

TABLE 4. Analysis of minimum, maximum and average $F 0$ values, in the sample of the sentence "Now it's time to finish", collected pre and post speech-language therapy of voice adequacy.

\begin{tabular}{|c|c|c|}
\hline Fundamental Frequency (Hz) & Pre-therapy & Post-therapy \\
\hline Minimum & 74 & 82 \\
\hline Maximum & 259 & 133 \\
\hline Average & 146 & 116 \\
\hline
\end{tabular}

TABLE 5. Analysis of minimum, maximum and average intensity values, in the sample of the sentence "Erica drank pear and blackberry juice", collected pre and post speech- language therapy of voice adequacy.

\begin{tabular}{|c|c|c|}
\hline Intensity (Db) & Pre-therapy & Post-therapy \\
\hline Minimum & 41 & 38 \\
\hline Maximum & 71 & 70 \\
\hline Average & 64 & 62 \\
\hline
\end{tabular}


The tables show, regarding the extraction of measures of intensity in the four sentences, before and after speech-language therapy, that the average intensity was already ideal before and remains after the therapy [12], both the minimum as the maximum intensity had a slight drop in the four samples after therapy.

The short-term parameter extracted was the measure of the duration of vowels in the sentences recorded before and after the therapy of voice adequacy, and the results are in the tables below:

TABLES 9-12 shows that, regarding the extraction of measures of duration of vowels in the four sentences, before and after the speechlanguage therapy, the duration of vowels after therapy decreased in the samples collected of the four sentences at the end of therapy. This result demonstrates that prosodic aspects have been modified with the treatment.

TABLE 6. Analysis of minimum, maximum and average intensity values, in the sample of the sentence "Sonia can dance samba alone", collected pre and post speech-language therapy of voice adequacy.

\begin{tabular}{|c|c|c|}
\hline Intensity (Db) & Pre-therapy & Post-therapy \\
\hline Minimum & 44 & 37 \\
\hline Maximum & 71 & 69 \\
\hline Average & 63 & 61 \\
\hline
\end{tabular}

TABLE 7. Analysis of minimum, maximum and average intensity values, in the sample of the sentence "Look at that blue airplane", collected pre and post speech-language therapy of voice adequacy.

\begin{tabular}{|c|c|c|}
\hline Intensity (Db) & Pre-Therapy & \multicolumn{2}{|c|}{ Post-Therapy } \\
\hline Minimum & 41 & 40 \\
\hline Maximum & 72 & 70 \\
\hline Average & 64 & 65 \\
\hline
\end{tabular}

TABLE 8. Analysis of minimum, maximum and average intensity values, in the sample of the sentence "Now it's time to finish", collected pre and post speech-language therapy of voice adequacy.

\begin{tabular}{|c|c|c|}
\hline Intensity (Db) & Pre-therapy & Post-therapy \\
\hline Minimum & 45 & 42 \\
\hline Maximum & 72 & 69 \\
\hline Average & 64 & 64 \\
\hline
\end{tabular}

TABLE 9. Measures of duration of vowels collected after phonetic segmentation of samples of the sentence "Erica drank pear and blackberry juice", pre and post speech- language therapy of voice adequacy.

\begin{tabular}{|c|c|c|c|}
\hline Pre vowels & Duration (Ms) & Post vowels & Duration (Ms) \\
\hline$[E ́]$ & 0.12 & {$[E ́]$} & 0.11 \\
\hline[] & 0.09 & {$[\mathrm{I}]$} & 0.04 \\
\hline$[\mathrm{A}]$ & 0.10 & {$[\mathrm{~A}]$} & 0.04 \\
\hline$[\mathrm{O}]$ & 0.08 & {$[\mathrm{O}]$} & 0.03 \\
\hline$[\mathrm{O}]$ & 0.11 & {$[\mathrm{O}]$} & 0.07 \\
\hline$[\mathrm{U}]$ & 0.05 & {$[\mathrm{U}]$} & 0.04 \\
\hline$[\mathrm{U}]$ & 0.04 & {$[\mathrm{U}]$} & 0.01 \\
\hline$[\mathrm{I}]$ & 0.04 & {$[\mathrm{I}]$} & 0.03 \\
\hline$[\hat{E}]$ & 0.17 & {$[\hat{E}]$} & 0.13 \\
\hline$[\mathrm{A}]$ & 0.11 & - & - \\
\hline$[\mathrm{la}]$ & 0.16 & {$[\mathrm{la}]$} & 0.15 \\
\hline$[$ [́] & 0.10 & {$[\mathrm{O}]$} & 0.15 \\
\hline$[\mathrm{A}]$ & 0.11 & {$[\mathrm{~A}]$} & 0.10 \\
\hline
\end{tabular}


TABLE 10. Measures of duration of vowels collected after phonetic segmentation of samples of the sentence "Sonia can dance samba alone", pre and post speech-language therapy of voice adequacy.

\begin{tabular}{|c|c|c|c|}
\hline Pre vowels & Duration (Ms) & Post vowels & Duration (Ms) \\
\hline$[\mathrm{O}]$ & 0.14 & {$[\mathrm{O}]$} & 0.11 \\
\hline$[\mathrm{E}]$ & 0.07 & {$[\mathrm{E}]$} & 0.07 \\
\hline$[\mathrm{A}]$ & 0.10 & {$[\mathrm{~A}]$} & 0.08 \\
\hline$[\tilde{\mathrm{A}}]$ & 0.13 & {$[\tilde{\mathrm{A}}]$} & 0.06 \\
\hline$[\mathrm{A}]$ & 0.15 & {$[\mathrm{~A}]$} & 0.07 \\
\hline$[$ Ó] & 0.11 & {$[\mathrm{Ó}]$} & 0.10 \\
\hline$[\mathrm{la}]$ & 0.19 & {$[\mathrm{la}]$} & 0.15 \\
\hline Total time of pre vowels & 0.89 & Total time of post vowels & 0.64 \\
\hline
\end{tabular}

TABLE 11. Measures of duration of vowels collected after phonetic segmentation of samples of the sentence "Look at the blue airplane", pre and post speech-language therapy of voice adequacy.

\begin{tabular}{|c|c|c|c|}
\hline Pre vowels & Duration $(\mathbf{M s})$ & Post vowels & Duration (Ms) \\
\hline$[\mathrm{O}]$ & 0.11 & {$[\mathrm{O}]$} & 0.07 \\
\hline$[\mathrm{A}]$ & 0.10 & {$[\mathrm{~A}]$} & 0.09 \\
\hline$[\mathrm{A}]$ & 0.18 & - & - \\
\hline$[\mathrm{Ua}]$ & 0.16 & {$[$ Aua] } & 0.30 \\
\hline$[\mathrm{lão}]$ & 0.30 & {$[$ lãoa] } & 0.32 \\
\hline$[\mathrm{A}]$ & 0.14 & - & - \\
\hline$[\mathrm{U}]$ & 0.13 & Total time of post vowels & 0.12 \\
\hline Total time of pre vowels & 1.12 & 0.90 \\
\hline
\end{tabular}

\begin{tabular}{|c|c|c|c|}
\hline Pre vowels & Duration (Ms) & Post vowels & Duration (Ms) \\
\hline$[\mathrm{A}]$ & 0.10 & {$[\mathrm{~A}]$} & 0.09 \\
\hline [Ó] & 0.27 & [Ó] & 0.19 \\
\hline$[\mathrm{A}]$ & 0.08 & - & \\
\hline [Eó] & 0.28 & [Eó] & 0.25 \\
\hline$[\mathrm{A}]$ & 0.12 & {$[\mathrm{~A}]$} & 0.05 \\
\hline [Ea] & 0.10 & [Ea] & 0.08 \\
\hline$[\mathrm{A}]$ & 0.05 & {$[\mathrm{~A}]$} & 0.04 \\
\hline$[\mathrm{A}]$ & 0.11 & {$[\mathrm{~A}]$} & 0.10 \\
\hline Total time of pre vowels & 1.11 & Total time of post vowels & 0.80 \\
\hline
\end{tabular}

\section{Discussion}

The vocal changes resulting from the process of voice adequacy promotes a better quality of life also related to psychological-emotional factors of the individual. The work of vocal readjustment in the life of an individual has a direct relationship with the degree of modification of the voice quality. Thus, measuring the degree of modification of the voice quality is sufficient to provide information on how the voice adequacy modifies people's quality of life. WellSucceeded vocal rehabilitation requires knowing the individual's vocal behavior. In this case, the whole structure of this voice does not reside in the vocal product, but rather in the laryngeal adjustment.

Observing the patient's vocal behavior is a functional strategy that provides better information on vocal behavior and brings a clearer idea of the reason for the individual not to accept his own voice. According to Schwarz et al. [13], this non-acceptance can refer to the fact that the male voices, when not treated, have an F0 focus changed. The changes most commonly found in the voice quality of transgender men have reduced the fundamental frequency with a low pitch, increased hoarseness/roughness, and the presence of instability [8].

The results of the auditory-perceptual evaluation presented in TABLE 13 show that the parameters of voice quality became closer to neutrality after the voice-adequacy therapy, mainly in relation to sound aspects. The auditory-perceptual analysis also shows that, after the therapy, the patient presented, an adjustment of the vocal tract, pharyngeal expansion, when, before the speech-language 
TABLE 13. Results of auditory-perceptive analysis performed using the VPAS-PB guide, pre and post-speechlanguage therapy of voice adequacy.

\begin{tabular}{|c|c|c|}
\hline Vpas-pb & Pre-therapy voice profile & Post-therapy voice profile \\
\hline Aspects of the vocal tract & $\begin{array}{c}1^{\text {st }} \text { degree decreased jaw Extension, } 1^{\text {st }} \text { degree vocal Tract and } \\
\text { Laryngeal hyperfunction, } 1^{\text {st }} \text { degree retreated tongue body }\end{array}$ & $1^{\text {st }}$ degree Pharyngeal expansion \\
\hline Sound aspects & Rough voice degree 1 & Modal voice \\
\hline Aspects of vocal dynamics & $1^{\text {st }}$ degree slow elocution rate & $\begin{array}{c}1^{\text {st }} \text { degree decreased pitch extension and } \\
\text { variability, } 1^{\text {st }} \text { degree fast elocution rate }\end{array}$ \\
\hline Vpas-pb & Pre-therapy voice profile & Post-therapy voice profile \\
\hline Aspects of the vocal tract & $\begin{array}{c}1^{\text {st }} \text { degree decreased jaw Extension, } 1^{\text {st }} \text { degree vocal Tract and } \\
\text { Laryngeal hyperfunction, } 1^{\text {st }} \text { degree retreated tongue body }\end{array}$ & $1^{\text {st }}$ degree Pharyngeal expansion \\
\hline Sound aspects & Rough voice degree 1 & $\begin{array}{c}\text { Modal voice } \\
\text { Aspects of vocal dynamics }\end{array}$ \\
\hline
\end{tabular}

therapy, showed decreased jaw extension, retreated tongue body and hyperfunction of the vocal tract. Unlike the result found in the study of Azul et al., the auditory-perceptual analysis did not reveal increased hoarseness/ roughness and the presence of instability. We believe that the difference in the results found are related to the importance of the speechlanguage therapy in the readjustment process, in which the transgender patient should not "find his ideal voice" doing any type of sound effort, but through the use of correct settings and intonation changes.

In this research, the long-term acoustic analysis, when analyzing the prosodic aspects of the speech of a trans man, found lower F0 values and difference between the maximum and the minimum F0 during the issuance of the sentences, corroborating the results found in Oates and Dacakis [14]. The shortterm acoustic analysis identified that, after the therapy, the patient began to produce shorter vowels, presenting a faster speech after speechlanguage therapy [13]. Under the perceptual point of view, this characteristic corresponds to the more directive character usually noticed in the speech of the transgender man.

In general, we found the consistency of findings in relation to the parameter intensity between the periods compared, occurring the permanence of the employment of medium intensity preserved [15]. We believe that the employment of an average intensity preserved by transgender man would be a way of balancing the vocal tissue. The patient's vocal complaint was not initially considered as a factor of inclusion/ exclusion in the present study. However, the analysis revealed that the element that guided his complaint, such as the "untuned voice", brought implications of emotional nature, which effectively made the patient defensive in his actions, with a high degree of inhibition. Future studies should be encouraged to better clarify this issue and provide greater knowledge of these aspects [8].

\section{Conclusion}

The acoustic measures of fundamental frequency demonstrate that after the speechlanguage therapy process, the patient presented less variation of melody, but his rhythmic variation was more quickly and with less duration time of the vowel, which gives more virile intonation characteristics to his speech. The intensity measures showed balanced values, which keeps the speech and voice with greater stability. Regarding the pre and post-therapy results, if they were just product of the use of the Durateston hormone, there would be only decreased pitch, since the drug promotes the enlargement of the larynx, but the results show changes in relation to the adjustments of the vocal tract and vocal dynamic, which stresses the importance of the speech-language therapy combined with the use of hormones for the success of the voice readjustment of the male transgender. 


\section{REFERENCES}

Lanz L. O corpo da roupa: a pessoa transgênera entre a conformidade $\mathrm{e}$ a transgressão das normas de gênero. Curitiba: Transgente. (2014).

Grossi MP, Ávila S. Transexuality and transgender movement in the perspective of the queer diaspora. Brazilian Journal of Legal Studies-Santo Agostinho College. 6(1), 155-170 (2017).

Nery JW, Maranhão EMDA. Trnsmen in cyberspace: micropolitics of resistances. In:Visibilidade Trans. 2, 139-165 (2013).

Lima F, Cruz KT. The harmonization process and the production of health care in male transsexuality. Sexualidad, Salud y Sociedad (Rio de Janeiro). 23, 162-186 (2016).

Athayde AV. Male transsexualism. Brazilian archives of endocrinology and metabolism. Arq Bras Endocrinol Metab. 45(4), 407-414 (2001).

Saadeh A. Sexual identity disorder: a psychopathological study of male-tofemale and female-to-male transsexuals Doctorate's Thesis. University of São Paulo. (2004).

Drumond LB, Gama ACG. Correlation between spectrographic and perceptual analysis of abnormal voices. Fono. Atual. 8(35), 49-58 (2006).

Azul D, Nygren U, Södersten M, Neuschaefer-R. Transmasculine people's voice function: A review of the currently available evidence. J. Voice. 31(2), 261 (2016).

Jacobs DDS. Vocal body, gender and performance. Rev. Bras. Estud. Presence. 7(2), 359-381 (2017).

Camargo Za. Analysis of the vocal quality of a group of dysphonic individuals: an interpretative and integrated approach of acoustic, perceptual and electroglotographic vocal data and poststroke dysphagia: acoustic, physiological and perceptual aspects. Dissertation. PUC/SP. São Paulo. 2002.
Boersma P, Weenink D. Praat: doing phonetics by computer. In: http://www. fon.hum.uva.nl/praat/. Amsterdan. (2002).

Behlau M, Azevedo R, Ponte P. Concept of normal voice and classification of dysphonia. Voice: the specialist's book. Rio de Janeiro: Revinter. 53-84 (2001).

Schwarz K, Fontanari AMV, Costa AB, et al. Perceptual-auditory and acoustical analysis of the voices of transgender women. Journal of Voice. 14(5), 266-267 (2017).

Oates J, Dacakis G. Transgender voice and communication: research evidence underpinning voice intervention for maleto-female transsexual women. Perspectives of the ASHA Special Interest Groups. 25, 48-55 (2015).

Avila SN. Ftm, transhuman, trans man, trans, man: The emergence of transmasculinities in contemporary Brazil. (2014)..

This special issue on Current Trends in Clinical Research was edited by Dr. Giuseppe Merra. 